\title{
Health as a key driver of climate change communication. A scoping review
}

\author{
Shukrullah Ahmadi ${ }^{1}$, Stefanie Schütte ${ }^{1}$, Niamh Herlihy ${ }^{1}$, Mathieu Hemono ${ }^{1}$, Antoine Flahault ${ }^{1,2}$ \\ and Anneliese Depoux ${ }^{1,3, *}$ \\ 1 Centre Virchow-Villermé for Public Health Paris-Berlin, Université Paris Descartes, Université Sorbonne \\ Paris Cité, France; shukrullah.ahmadi11@gmail.com; stefanie.schutte@parisdescartes.fr; \\ Niamh.herlihy@gmail.com; mhemono@bu.edu; \\ 2 Institute of Global Health, Faculty of Medicine, University of Geneva, Switzerland; \\ antoine.flahault@unige.ch \\ 3 3GRIPIC, EA 1498, Université Paris Sorbonne - CELSA, France \\ * Correspondence: anneliese.depoux@uspc.fr; Tel.: +33-(0) 06-6182-0817
}

\begin{abstract}
The negative implications of climate change for human health are now well established. Yet these have not been fully considered into climate change communication strategies. Research suggests that reorienting climate change communication with a health frame could be a useful communication strategy. We conducted a long-term and broad overview of existing scientific literature in order to summarize the state of research activity in this area. The methodology is based on a scoping review of scientific articles published on climate change communication and health between 1990 and mid-2016 indexed in the PubMed, ScienceDirect, and Web of Science databases. The screened citations were reviewed for inclusion and data were extracted in order to conduct quantitative (e.g. frequencies) and qualitative (i.e. content analysis) analyses. Out of 2,866 identified published papers, only 24 articles were eligible for analyses. The main categories identified were reframing climate change as a health issue $(n=10,41.7 \%)$, the role of health professionals $(n=10$, $41.7 \%)$ and the perception of climate change $(n=4,16.6 \%)$. We identified a large proportion of secondary research articles $(n=15,62.5 \%)$ including reviews $(n=5,20.8 \%)$ and opinion articles $(n=10$, $41.7 \%)$. A significant share $-37.57 \%(\mathrm{n}=9)$ - of the identified articles were classified as original research articles, suggesting that the number of publications in this area - particularly original research - has not grown rapidly. This scoping review identified several categories including reframing climate change as a health issue, the role of health professionals, and the perception of climate change in the selected articles on the subject. The research literature on the communication of climate change and health is relatively recent and emerging.
\end{abstract}

Keywords: climate change communication; health; public engagement; health frame

\section{Introduction}

As early as 1990, the World Health Organization (WHO) and the Intergovernmental Panel on Climate Change (IPCC) identified significant health implications of climate change [1, 2]. According to conclusions of the IPCC, the implications are not only worrying for the environment but also for human health [3]. Extreme weather conditions, poor air quality, and varying patterns of vector-borne diseases influence global health [4-8]. This makes climate change one of the most evolving and emerging threats to the health of populations globally $[9,10]$. Despite this threat, it has been consistently reported that the health effects of climate change are not well understood in both public and policy discourses [11]. Nevertheless, health as a key driver of climate change communication has recently received a growing attention from researchers interested in climate change and health [12, 13].

"Communication is be defined as the production and exchange of information to inform, influence, or motivate an individual, institutional, and public audiences" [14]. Communication can be used to bring together scientists, policymakers and the public, and therefore it should enable public engagement with climate change [15]. For instance, it has been shown that the health argument 
is increasingly embedded in policy debates. COP 21 was an obvious example of this as for the first time 'the right to health' was mentioned in the global agreement on climate change [16]. In addition, the role of mass media in raising awareness on the health impacts of climate change is growing [5, 17]. However, research has stressed that mass media is not sufficiently utilized to bring about behavioral change and engagement among the public [18]. According to surveys on the public understanding of climate change, there is still a great lack of a comprehensive understanding of climate change among the public [18]. Recent studies have shown that reorienting climate change communication with a positive public health frame may provide efficient engagement and behavioral change among the public $[12,13]$.

For almost three decades, climate change communication has been an emerging issue [19] but unfortunately it has not relied on a strong basis of scientific evidence [15]. Studies have reported that there is limited evidence linking climate change, public health and behavioral change [13]. However, the amount and type of research literature linking climate change communication and health is not well known. Considering the Lancet Commission's call for further research on public health and climate change [20], we conducted a scoping review of the current published literature on Climate Change Communication and Health (henceforth abbreviated as CCC\&H).

\section{Research objectives and questions}

The objectives of this scoping literature review were (1) to provide an overview of existing scientific articles over time regarding $\mathrm{CCC} \& \mathrm{H} ;(2)$ and to describe the scope (i.e., amount, focus and nature) of existing scientific articles on CCC\&H. Therefore, we sought to answer the following research questions:

1. To what extent is the subject covered in the scientific literature between 1990 and mid-2016.

2. What is the geographical focus of the publications?

3. What types of articles (e.g. reviews, original research) are published on the topic?

\section{Materials and Methods}

This review followed the scoping review framework proposed by Arksey and O'Malley [21]. An important strength of the scoping review method, as applied to this review, is that it allows for the analysis of a broad research question [22]. Climate change communication and health is a vast area of research and our aim was to map the extent of the research activity on this topic. Hence, we reviewed published scientific articles from 1990 to mid-2016 in order to give a broad overview on this topic and explore research gaps and trends [21]. 1990 was chosen as the starting year for this study, as it is the year of publication of the first IPCC report. We mapped our findings by classifying literature according to several categories identified in the analysis phase.

\subsection{Identification of relevant studies and search strategy}

One reviewer searched three main databases: ScienceDirect, Pubmed, and Web of Science. All three databases were chosen as they include multidisciplinary literature (ScienceDirect and Web of Science), in particular literature from environmental sciences, social sciences and health sciences. The PubMed database helped us access literature from domains such as medical sciences, life sciences, and social sciences. Databases were searched in May and June 2016.

The search strategy combined related search terms of climate change, communication and health. The search strategy, in summary, included ("Climate change" OR "Global warming" OR "Greenhouse effect") AND (Communicat* OR Frame* OR Engage OR Media OR Behaviour Change) AND (Health OR "Public health" OR "Global Health"). Where relevant, a wildcard search term (e.g. "communicat") was used so that it covers "communication" or "communicating". EndNote X7 reference management software was used for managing and screening citations obtained from the search strategy. 


\subsection{Study selection}

We included all articles published in English, including original quantitative and qualitative studies, reviews, editorials, and viewpoints. We only included literature which mentioned the search terms listed above in their keywords, title, or abstract. We did not include book chapters or grey literature (dissertations, conference proceedings, thesis, reports etc.). We also excluded papers that had no focus on climate change communication or lacked focus on human health (figure 1). The collected articles were screened by one reviewer (SA) and verified by another reviewer (NH) for relevance against the inclusion and exclusion criteria. The papers that did not meet the inclusion criteria were excluded. A final selection was made upon a final reading of the full-texts (figure 1). The two reviewers (SA and $\mathrm{NH}$ ) met several times during the abstract selection process to discuss agreements or disagreements, challenges faced, and uncertainties related to the selection of the papers. We went back and refined the search strategy as needed. The remaining team (AD, SC, MH) assisted the first reviewers with conflict resolution and whenever it was necessary in case of disagreement.
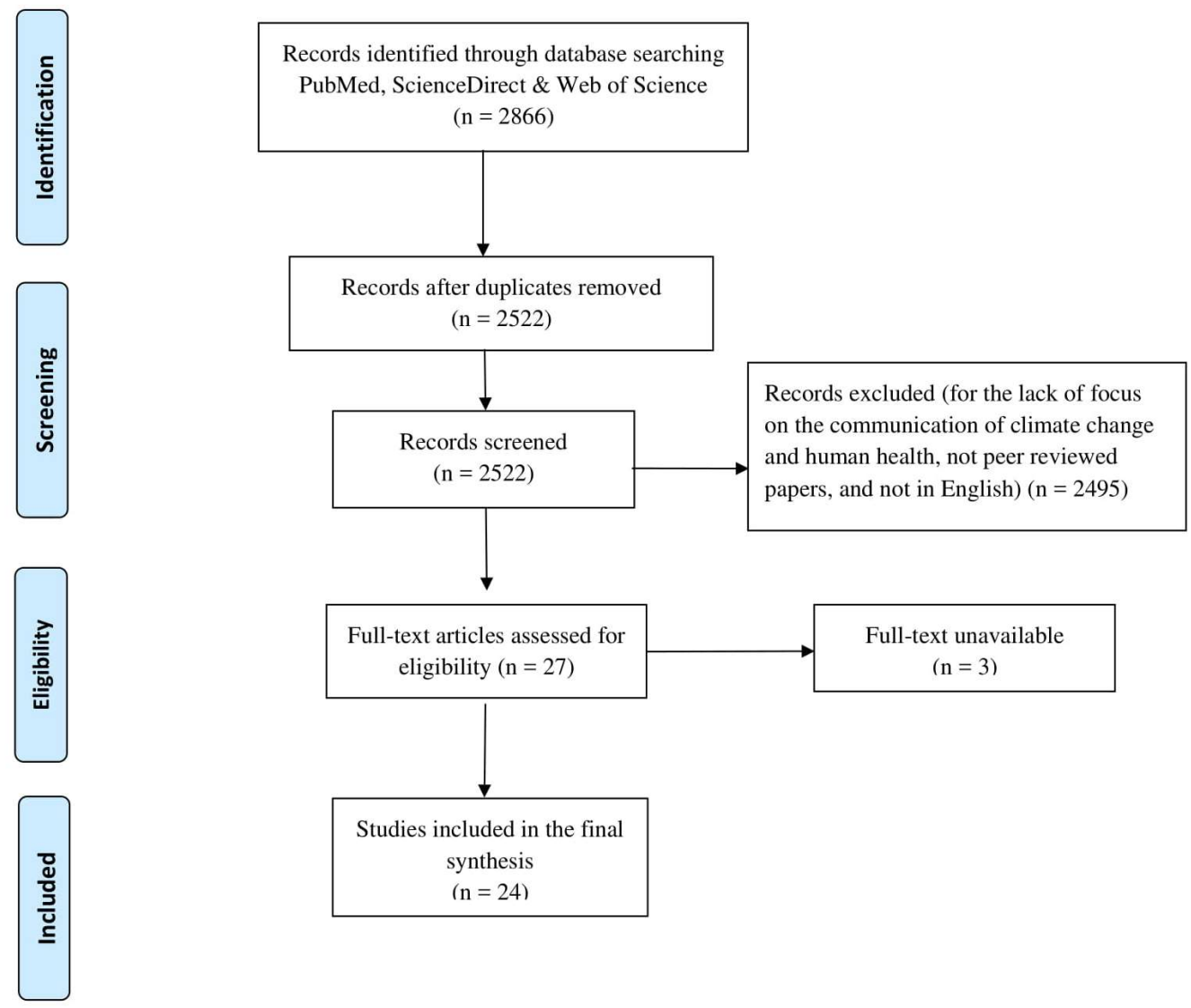

Figure 1. Flowchart of literature search and screening

\subsection{Charting, collating and summarizing data}

We used a qualitative meta-summary to summarize the results following data extraction, grouping, abstraction, and calculation of frequencies [23]. A descriptive summary was provided that included the following information from the included articles: author/s, publication year, journal, study type (original research article, original review, review, viewpoint), geographic region of the study, and category of each article. These categories (reframing climate change as a health issue, the role of health professional, and the perception of climate change) were assigned through an iterative process and were created during the article reading. The extracted data was extrapolated into a data- 
charting form in a Microsoft Excel 2013 (Microsoft Corporation, Redmond, WA). One reviewer extracted the data using the data-charting form and met with the research team (NH, AD, SC) to determine whether the data extraction approach was consistent with the research question and purpose.

\section{Results}

\subsection{Study characteristics}

Figure 1 presents a flowchart that summarizes the results of the search strategy and the article selection processes. Of the 2,866 articles identified through the various database searches, 24 articles were selected for the final review. The detailed list of 24 articles are presented in the supplementary table S1. Table 1 presents characteristics of the included articles while Table 2 lists the three categories of articles included in this review. These are the only categories we came up with and they are not examples of a larger grouping.

The earliest published articles were out in 2008 (Table 1). Since then a sharp increase has been observed with maximum seven articles published in 2014 and 12 articles (52\%) published between 2014 and mid-2016.

$37.5 \%$ of the articles $(n=9)$ were published as original research articles and the majority used cross-sectional methods such as surveys and interviews $(n=9)$ while one study used a randomized controlled message experiment. More than half of the articles $(62.5 \%, n=15)$ were secondary research articles including literature reviews and opinion articles. Among the literature review articles, none of the articles were systematic reviews.

Table 1. Characteristics of the included articles.

\begin{tabular}{|c|c|c|}
\hline Characteristics & n (\%) & References \\
\hline \multicolumn{3}{|l|}{ Publication year } \\
\hline 2008 & $2(8.3)$ & {$[14,24]$} \\
\hline 2009 & 0 & \\
\hline 2010 & $4(16.7)$ & {$[12,25-27]$} \\
\hline 2011 & $1(4.2)$ & [28] \\
\hline 2012 & $2(8.3)$ & {$[29,30]$} \\
\hline 2013 & $3(12.5)$ & {$[13,31,32]$} \\
\hline 2014 & $7(29)$ & {$[11,33-38]$} \\
\hline 2015 & $3(12.5)$ & [39-41] \\
\hline 2016 & $2(8.3)$ & {$[42,43]$} \\
\hline \multicolumn{3}{|l|}{ Types of articles } \\
\hline Original research & $9(37.5)$ & {$[12,13,25,26,28,29,36,38,40]$} \\
\hline Review & $5(20.8)$ & {$[11,30,32,33,37]$} \\
\hline Viewpoint & $10(41.7)$ & {$[14,24,27,31,34,35,39,41-43]$} \\
\hline \multicolumn{3}{|c|}{ Geographical focus } \\
\hline Africa & 1 & {$[36]$} \\
\hline North America & 8 & {$[12-14,28,29,33,37,40]$} \\
\hline \multicolumn{3}{|l|}{ South America } \\
\hline Asia & 1 & {$[38]$} \\
\hline Europe & 3 & {$[26,30,31]$} \\
\hline Not given/NA ${ }^{1}$ & 10 & {$[11,24,27,32,34,35,39,41-43]$} \\
\hline Multiple regions & 1 & {$[25]$} \\
\hline
\end{tabular}

${ }^{1}$ Not applicable 
The geographical focus of the articles varied, however, more than half of the articles were based in upper-middle and high-income countries $(n=13)$, more than half of which were original research articles. Five original research articles had a geographical focus in the United States (US), two in the United Kingdom (UK), one in Canada, one in Malta, and one original research article focused on three countries together (Malta, Canada, and US). One study explored the issue in China and one in Ethiopia, both being original research articles. Ten articles that were not original research, but mainly literature reviews, did not have a specific geographical focus.

Ten articles (10/24, 41.7\%) mentioned framing and/or reframing climate change as a health or public health issue. While ten articles $(10 / 24,41.7 \%)$ described the role of health professionals (e.g. clinicians, general practitioners, and nurses) and other public health professionals in communicating the risk of climate change to different audiences such as patients and the general public. Four studies assessed knowledge, practices, and perceptions of the public about climate change as a health issue.

Table 2. Categories of the included articles

\begin{tabular}{lll}
\hline Categories & $\mathrm{n}(\%)$ & References \\
\hline $\begin{array}{l}\text { Reframing climate change } \\
\text { as a health issue }\end{array}$ & $10(41.7)$ & {$[12,14,24,28,29,32-35,39]$} \\
$\begin{array}{l}\text { The role of health } \\
\text { professionals }\end{array}$ & $10(41.7)$ & {$[11,27,30,31,36,37,40-43]$} \\
$\begin{array}{l}\text { The perception of climate } \\
\text { change }\end{array}$ & $4(16.6)$ & {$[13,25,26,38]$} \\
\hline
\end{tabular}

\subsection{Reframing climate change as a health issue}

All the ten-identified articles in this category highlighted the need for effective communication of climate change, which could help to effectively reach people, change their behavior, attitudes and perception of climate change. Articles outlined the attributes and strategies of effective CCC\&H. The articles grouped in this category described some major crosscutting attributes and strategies such as framing climate change with a health message.

Seven studies [11, 12, 28, 29, 33-35] highlighted communicating the message of climate change with a health frame. Reframing climate change as a public health issue makes the problem personal, significant, relevant and understandable. Three original research articles [12, 24, 28] from the United States studied public reactions towards framing climate change as a health issue. These studies strongly addressed that health frame can be used to complement other strategies in order to enhance the public engagement towards mitigation and adaptation behaviors [12, 29, 34, 35, 41]. Such a frame promotes health as a co-benefit among other benefits (environmental, economic and social) of actions that address climate change $[12,24,28,29,34,35,39]$. The literature we reviewed linked different benefits mainly health benefits attainable through climate change mitigation. Three studies described the significance of positive messages while communicating climate change [14, 24, 39]. Effectively communicating climate change requires positive messages that promotes "constructive engagement" of public towards climate change [15, 44]. Alarming messages such as promoting fear and despair might not be effective. Two papers focused on further strategies to make communication effective including consideration of individual values and motivation and use of emotions [14, 39].

\subsection{The role of health professionals in $\mathrm{CCC \& H}$}

We identified ten articles pertinent to the role of health professionals in CCC\&H. Many articles $(n=6 / 10)$ in this category addressed the crucial role of health professionals as messengers in communicating and addressing climate change as a health issue [11, 24, 27, 39, 40, 43]. Their role is also highlighted in the public discourse, advocacy, and policy formation around mitigating the impacts of climate change and reducing emissions. For example, health professionals could accurately communicate information to the public and to policymakers about the health impacts of emissions and fossil fuel policies and on the importance of energy efficiency and cleaner energy 
production to reduce greenhouse gas emissions [11]. Doctors and nurses were the main healthcare professionals described in the literature we reviewed $(n=5)$.

One paper addressed the role of clinicians and the healthcare sector in reducing the sector's greenhouse gas emissions in different aspects of healthcare (transportation, energy conservation and efficiency, waste management, nutrition services and water conservation) [27]. One of the articles partly focused on the role of health communication professionals in communicating health effects of climate change [42]. Two main roles defined are understanding the communication process and supporting health systems in addressing the health effects of climate change. One of the studies also addressed the need of interdisciplinary collaboration between health and environmental education professionals and highlighted the opportunity for environmental practitioners to benefit from the developments in the health profession and health education in order to address the risk of climate change effectively [11]. One of the examples of collaborative work suggested, in relation to communication of climate change, was reframing climate change as a health problem along with the presentation of health co-benefits of mitigation. The authors also partly described the reasons of why healthcare professionals should be concerned about climate change. The most common given reason identified in these articles was the possession of required knowledge and experience by the healthcare professionals [11, 27, 39]. Another two studies in this category added that healthcare professionals are the most trusted experts in the field within the context of their role in educating patients and their families [27, 31].

Many studies suggested several ways and actions health professionals should take in response to climate change such as supporting policy makers in mitigation and adaptation and taking part in strategies to reduce greenhouse gas emissions and reduce energy consumption [43]. Several individual actions (advise patients to walk or bike instead of driving, convey health impacts of climate change) and collective actions (general messages that climate is not a distant problem, and convey the risks of climate change to vulnerable groups i.e. children, elderly, poor, diseased and some races) advised by health professionals are described in the literature [39, 43]. Providing information on climate change related diseases to patients (e.g. asthma) is also mentioned [43].

Several studies $(n=5)$ identified the need for more training among health professionals with regard to communication and engagement towards the health effects of climate change [11, 27, 30, $36,40]$. We found some articles that addressed the gaps in the awareness and knowledge among health professions about climate and its health effects. Another similar original research article explored knowledge about the health impacts of climate change among health science students in Ethiopia and found that $87.9 \%$ of students reported lack of essential knowledge on how to address health impacts of climate change [36]. This study highlighted that information received through mass communication is not sufficient and emphasized on improving the university curriculum. Similarly, another review article addressed the need for further education on climate change for sustainability initiatives in other health professions and in nursing profession by improving nursing curriculums [30]. Another review focused on preparing nurses including nurse leaders and academicians to better respond to the public health impacts of climate change by presenting several informative ways of engagement through advocacy and policy involvement [11].

\subsection{The perception of climate change}

Articles categorized in this category mainly reflected the benefits of framing climate change with a health message. We found four original cross-sectional studies that assessed knowledge, practices, and/or perceptions about climate change. One study carried out an international comparison in USA, Canada, and Malta [25] while the second and third study had a single-site methodology in Canada [13] and Malta [26] respectively. The studies reported that individuals who perceive climate change as a threat to human health and well-being can be supportive towards climate change mitigation policies, therefore, framing climate change as a health problem can be effective in gaining public support and promoting public engagement $[13,25,26]$. Another research study studied perception and knowledge gaps among the Chinese Center for Diseases Control (CDC) health professional in 
China and recommended for more efforts to be made in order to raise awareness and enhance knowledge among health professions [38].

\section{Discussion}

Based on our scoping review and the content analysis, 24 relevant articles and 3 major categories were identified: 1) reframing climate change as a health issue 2) the role of health professionals in CCC\&H and 3) the perception of climate change. The first article was published in 2008 and since then a sharp increase in the number of publications are observed. Ten publications were original research articles and most articles focused on upper-middle and high-income countries. We found in the selected articles that traditional presentation of the issue of climate change such as presenting it as an environmental issue may not be useful in bringing about low carbon and low greenhouse gas emission behaviors among public and decision makers.

Research on CCC\&H is relatively recent and emerging as the first publication on this topic appeared in 2008. It has been reported that the overall trend of scientific publications on climate change and health increased rapidly particularly between 2008 and 2009 [5]. The published literature we reviewed suggested that communicating climate change framed with a health argument could be effective to a variety of audiences including public and decision makers. They also addressed the need, significance, and strategies of communicating climate change with a health frame. The literature also frequently highlighted that climate change has been traditionally presented as an environmental, distant, or a national security problem which is one of the constraints of effective climate change communication because these methods of issue presentation have not been successful enough in bringing public engagement. As a result, this has led to a lack of motivation, interest, and "issue fatigue" among the public [12]. Therefore, it is high time that we adapt our communication strategies and make it more effective by using a health frame when communicating about climate change. When climate change is communicate through a health frame, it becomes personal, significant and relevant.

However, there is a significant lack of empirical evidence for specific strategies [32]. For example, many studies addressed the significant role of health professionals in this regard through their role in education, public discourse, advocacy, and policy. However, we found limited amount of original research in this area.

The published literature on CCC\&H has a diverse focus and articles have mostly a mixed approach linking CCC\&H to other disciplines such as marketing, environmental education, health communication, psychology (risk and perception) and bioethics sciences demonstrating the complexity of communication and the need for more interdisciplinary collaboration and research.

Considering the fact that we found the majority of these articles including original research articles were published in upper-middle and high-income countries, more research would be appreciated in low and lower-middle income countries who will mostly face the health impacts of climate change. Future studies could study the risk perception of public, preparedness of health professionals on communicating health impacts of climate change with a special focus in low and lower-middle income countries.

This was our first encounter as a research team identifying literature on CCC\&H with the scoping review methodology and presenting the results with a more qualitative approach. Scoping method has been embraced by several research and granting organizations as a method for mapping and synthesizing existing literature in a particular topic area and identifying gaps where future research should be conducted. We believe that this double approach (scoping with an in-depth qualitative analyses) is a strength of our study.

However, several limitations should be considered when interpreting our results. We used only three databases, which may be a limitation even though all three databases are complementary and cover together the majority of the scientific publications in the area of climate change and human health. Moreover, grey literature is not captured in this review. Furthermore, we did not aim to review communication around other specific aspects of climate change such as communication around specific health impacts of climate change or on early warning systems or their variants. 
Therefore, additional search terms to cover these areas of climate change were not included in the search strategy. Also, the restriction to publications in English language only may be a caveat. Finally, the quality of identified scientific publications are not assessed which is a limitation of scoping reviews. Despite these limitation, to our knowledge this is review is one of the first of its kind that takes a first step towards organizing and summarizing this increasingly important body of literature.

\section{Conclusions}

The field of CCC\&H is relatively recent and emerging without a strong foundation of research. Research activity on CCC\&H appears to be increasing from 2008 onwards. It is getting the attention of those interested in promoting public engagement and reducing its health impacts. This first-ever scoping review on CCC\&H identified the state of research production by taking a long-term approach and reported several categories identified in the published literature on CCC\&H. To date, several cross-sectional and few experimental studies have been carried out that have investigated public and health professionals' knowledge and attitudes; and few tested the effectiveness of framing climate change as a health issue and relevant audience responses in various countries. In addition, more research, particularly from lower income countries is needed.

Supplementary Materials: Table S1: List of included publications on CCC\&H.

Author Contributions: Anneliese Depoux, Stefanie Schütte and Niamh Herlihy conceived and planned the review. Shukrullah Ahmadi, Niamh Herlihy and Mathieu Hemono carried out the search and screening of the literature. Anneliese Depoux, Stefanie Schütte and Shukrullah Ahmadi analyzed the data. Shukrullah Ahmadi drafted the review. Anneliese Depoux and Antoine Flahault supervised and provided administrative support. All authors revised the article critically for important intellectual content. All authors commented on, approved the final manuscript, and are accountable for all aspects of the work.

Funding: This research received no external funding

Conflicts of Interest: The authors declare no conflict of interest

\section{References}

1. Houghton, J.T., G.J. Jenkins, and J.J. Ephraums, Climate change: the IPCC scientific assessment. 1990: UK.

2. Organization, W.H., Potential effects of climatic change. 1990, World Health Organization: Geneva.

3. Woodward, A., et al., Climate change and health: on the latest IPCC report. Lancet, 2014. 383(9924): p. 11859.

4. Costello, A., H. Montgomery, and N. Watts, Climate change: the challenge for healthcare professionals. BMJ, 2013. 347: p. f6060.

5. Watts, N., et al., The Lancet Countdown on health and climate change: from 25 years of inaction to a global transformation for public health. Lancet, 2018. 391(10120): p. 581-630.

6. Haines, A., P.R. Epstein, and A.J. McMichael, Global health watch: monitoring impacts of environmental change. Lancet, 1993. 342(8885): p. 1464-9.

7. Haines, A., et al., Climate change and human health: impacts, vulnerability and public health. Public Health, 2006. 120(7): p. 585-96.

8. McMichael, A.J., R.E. Woodruff, and S. Hales, Climate change and human health: present and future risks. Lancet, 2006. 367(9513): p. 859-69.

9. Haines, A., et al., Public health benefits of strategies to reduce greenhouse-gas emissions: overview and implications for policy makers. The Lancet, 2009. 374(9707): p. 2104-2114.

10. Whitmee, S., et al., Safeguarding human health in the Anthropocene epoch: report of The Rockefeller Foundation-Lancet Commission on planetary health. Lancet, 2015. 386(10007): p. 1973-2028.

11. Adlong, W. and E. Dietsch, Environmental education and the health professions: framing climate change as a health issue. Environmental Education Research, 2014. 21(5): p. 687-709.

12. Maibach, E.W., et al., Reframing climate change as a public health issue: an exploratory study of public reactions. BMC Public Health, 2010. 10: p. 299.

13. Cardwell, F.S. and S.J. Elliott, Making the links: do we connect climate change with health? A qualitative case study from Canada. BMC Public Health, 2013. 13: p. 208. 
14. Maibach, E.W., C. Roser-Renouf, and A. Leiserowitz, Communication and marketing as climate changeintervention assets a public health perspective. Am J Prev Med, 2008. 35(5): p. 488-500.

15. Moser, S.C., Communicating climate change: history, challenges, process and future directions. Wiley Interdisciplinary Reviews: Climate Change, 2010. 1(1): p. 31-53.

16. Schutte, S., et al., The influence of health concerns in scientific and policy debates on climate change. J Epidemiol Community Health, 2017. 71(8): p. 747-749.

17. Depoux, A., et al., Communicating climate change and health in the media, in Public Health Rev. 2017.

18. Stamm, K.R., F. Clark, and P.R. Eblacas, Mass communication and public understanding of environmental problems: the case of global warming. Public understanding of science, 2000. 9(3): p. 219-237.

19. Nerlich, B., N. Koteyko, and B. Brown, Theory and language of climate change communication. Wiley Interdisciplinary Reviews: Climate Change, 2010. 1(1): p. 97-110.

20. Watts, N., et al., Health and climate change: policy responses to protect public health. Lancet, 2015. 386(10006): p. 1861-914.

21. Arksey, H. and L. O'Malley, Scoping studies: towards a methodological framework. International journal of social research methodology, 2005. 8(1): p. 19-32.

22. Herlihy, N., et al., Climate change and human health: what are the research trends? A scoping review protocol. BMJ Open, 2016. 6(12).

23. Sandelowski, M., J. Barroso, and C.I. Voils, Using Qualitative Metasummary to Synthesize Qualitative and Quantitative Descriptive Findings. Res Nurs Health, 2007. 30(1): p. 99-111.

24. Frumkin, H. and A.J. McMichael, Climate change and public health: thinking, communicating, acting. Am J Prev Med, 2008. 35(5): p. 403-10.

25. Akerlof, K., et al., Public perceptions of climate change as a human health risk: surveys of the United States, Canada and Malta. Int J Environ Res Public Health, 2010. 7(6): p. 2559-606.

26. DeBono, R., K. Vincenti, and N. Calleja, Risk communication: climate change as a human-health threat, a survey of public perceptions in Malta. Eur J Public Health, 2012. 22(1): p. 144-9.

27. Sayre, L., et al., Climate change and human health: the role of nurses in confronting the issue. Nurs Adm Q, 2010.34(4): p. 334-42.

28. Semenza, J.C., G.B. Ploubidis, and L.A. George, Climate change and climate variability: personal motivation for adaptation and mitigation. Environ Health, 2011. 10: p. 46.

29. Myers, T.A., et al., A public health frame arouses hopeful emotions about climate change. Climatic Change, 2012. 113(3-4): p. 1105-1112.

30. Barna, S., B. Goodman, and F. Mortimer, The health effects of climate change: what does a nurse need to know? Nurse Educ Today, 2012. 32(7): p. 765-71.

31. Johnson, G.A., Role of GPs in communicating lifestyle messages that are good for health and the climate. BMJ, 2013. 347: p. f6480.

32. Bostrom, A., G. Böhm, and R.E. O'Connor, Targeting and tailoring climate change communications. Wiley Interdisciplinary Reviews: Climate Change, 2013. 4(5): p. 447-455.

33. DeNicola, E. and P.R. Subramaniam, Environmental attitudes and political partisanship. Public Health, 2014. 128(5): p. 404-9.

34. Humphreys, G., Reframing climate change as a health issue. Bull World Health Organ, 2014. 92(8): p. 5512.

35. Valles, S.A., Bioethics and the Framing of Climate Change's Health Risks. Bioethics, 2015. 29(5): p. 334-41.

36. Nigatu, A.S., B.O. Asamoah, and H. Kloos, Knowledge and perceptions about the health impact of climate change among health sciences students in Ethiopia: a cross-sectional study. BMC Public Health, 2014. 14: p. 587.

37. Patz, J.A., et al., Climate change: challenges and opportunities for global health. JAMA, 2014. 312(15): p. 1565-80.

38. Wei, J., et al., Perception, attitude and behavior in relation to climate change: a survey among CDC health professionals in Shanxi province, China. Environ Res, 2014. 134: p. 301-8.

39. Ring, W., Inspire Hope, Not Fear: Communicating Effectively About Climate Change and Health. Ann Glob Health, 2015. 81(3): p. 410-5.

40. Gould, S. and L. Rudolph, Challenges and Opportunities for Advancing Work on Climate Change and Public Health. Int J Environ Res Public Health, 2015. 12(12): p. 15649-72. 


\section{0 of 10}

41. Adlong, W. and E. Dietsch, Nursing and climate change: An emerging connection. Collegian, 2015. 22(1): p. 19-24.

42. Chadwick, A.E., Climate Change, Health, and Communication: A Primer. Health Commun, 2016. 31(6): p. 782-5.

43. Koh, H., Communicating the Health Effects of Climate Change. JAMA, 2016. 315(3): p. 239-40.

44. Priest, E.M. and H. Susanna, No More "Business as Usual": Addressing Climate Change Through Constructive Engagement. http://dx.doi.org/10.1177/1075547008329202, 2009. 